Check for updates

Cite this: RSC Adv., 2017, 7, 47380

Received 16th June 2017

Accepted 3rd October 2017

DOI: $10.1039 / \mathrm{c} 7 \mathrm{ra06743g}$

rsc.li/rsc-advances

\section{Mussel-inspired hydrogel tissue adhesives for wound closure}

\author{
Maedeh Rahimnejad and Wen Zhong (D)* \\ Tissue adhesives have been introduced as a promising alternative for the traditional wound closure method \\ of suturing. Design and development of tissue adhesives for biomedical applications has been inspired by \\ outstanding examples in nature. This review covers the adhesive mechanisms, applications and \\ characterizations of various biomimetic tissue adhesives reported during the past decade, with a focus \\ on the mussel-inspired dopamine-based adhesives, which have attracted extensive interest due to their \\ promising adhesive performance in a wet environment.
}

\section{Introduction}

The advent of different wound closure methods has provided promising alternatives for such traditional wound closure methods as suturing. The knot tying procedure for sutures may cause tissue distortion, residual force, blocking of blood perfusion, and consequently impede wound healing. Foreign body reaction is another adverse outcome of sutures, especially at a site of knotting which represents a major mass of foreign body.$^{1-6} \mathrm{~A}$ primary closure of wounds, suturing sometimes may not be feasible because of the inadequacy of the skin flaps, especially after trauma or following excision of lesions. ${ }^{1}$ In this regard, alternative methods for surgical wound closure such as biological glues and tissue adhesives have gained more attentions recently. Tissue adhesives offer various advantages over traditional wound closure methods include easiness to apply, causing less pain and no need for removal in a further visit.

Tissue adhesives are becoming a common adjunct in surgical practice and are applied in tissue adhesion, hemostasis, and sealing of air and body fluids leakage during surgical procedure. Both biologic adhesives (e.g. fibrin-based adhesives) and synthetic adhesives (e.g. cyanoacrylate derivatives) are available for such applications. ${ }^{7,8}$ Comparing tissue adhesives and other wound closure techniques has been studied to determine different outcomes including cosmetic effect in the under study population..$^{9-11}$ For cosmetic reasons, tissue adhesives have gained increasing popularity as an alternative method of wound closure. ${ }^{12,13}$ In recent years, tissue adhesives have commercialized with significant advantages such as decreasing foreign body reaction during wound healing procedure and no need for further removal. ${ }^{14,15}$ Tissue adhesive systems have been used in dentistry, ${ }^{16-18}$ orthopedics, ${ }^{19-21}$ cardiovascular ${ }^{22,23}$ as well as in ophthalmology ${ }^{24}$ traumatology,

Department of Biosystem Engineering, University of Manitoba, Canada. E-mail: wen. zhong@umanitoba.ca and otorhinolaryngology and also for plastic and maxillofacial surgical applications. ${ }^{25,26}$ The advantages of hard tissue (e.g. bone or dent) gluing as compared to nailing or plating includes good fixation of small fragments, more homogenous weight bearing distribution within the fracture site. In the treatment of articular fractures, tissue adhesives act as subchondral spacer to compensate joint surface displacement. ${ }^{27}$ The applications of adhesives in soft tissues include skin $^{12}$ and ocular wounds closures. $^{28}$ Adhesion is critical in hemostatic biomaterials, therefore tissue adhesives play an important role to improve conventional sealing methods ${ }^{22}$ (Fig. 1). Chen et al. reported a mussel inspired hydrogel adhesive composed of $\operatorname{poly}(\gamma$-glutamic acid) and dopamine, which showed $41.2 \%$ reduction in bleeding as compared to fibrin glue. ${ }^{29}$

Researchers have also become interested in using tissue adhesives in other areas, such as artificial soft tissues and controlled drug and bioagents delivery systems for releasing

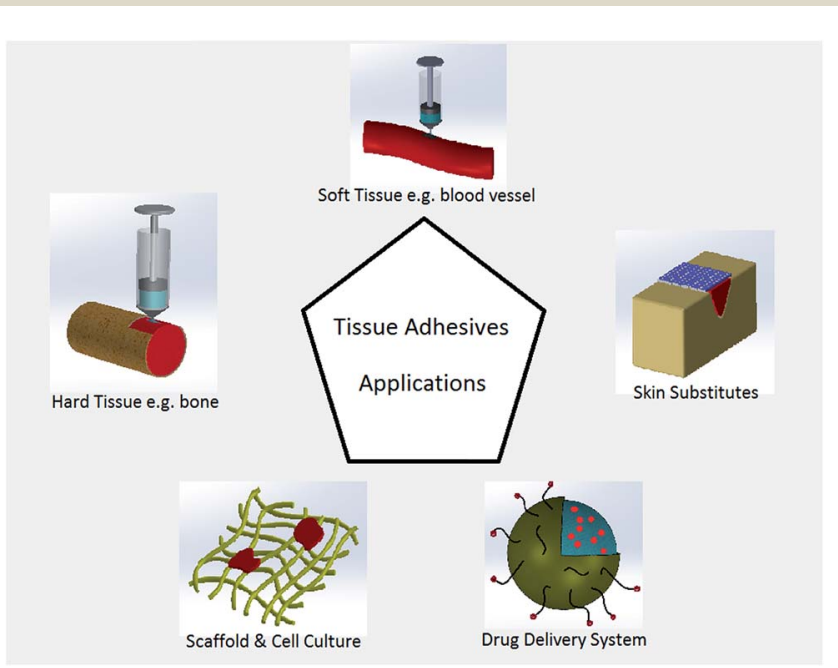

Fig. 1 Applications of tissue adhesives in biomedical engineering field. 
antibiotic and antibacterial drugs in the buccal or nasal cavity, for intestinal or rectal delivery, and even for application in urinary bladder. ${ }^{30-32}$ Injectable tissue adhesives loaded with cells can be a promising technique in cartilage impairment treatments. ${ }^{27}$ Tissue adhesives also showed appealing properties and functionality in cell culture systems and scaffolds modifications for appropriate cells responses, such as biospecific cell adhesion for better cell migration and viability. $\mathrm{Ku}$ et al. modified a microchannel by poly(dopamine) (PDA) as a simple and effective approach to maintain cell morphology in tissue engineering. ${ }^{33}$ Dopamine was also used as a coating material on another material like poly(ethylene glycol) to prepare a surface with better cell adhesive ${ }^{34}$ (Fig. 1).

Design and development of high-performance and environmentally friendly tissue adhesives has been inspired by many outstanding and promising examples in nature. One good example is the dopamine-based tissue adhesives inspired by the marine mussels that have the capacity to adhere to wet or moisture surfaces, which is an essential requirement for any adhesives to be used in the field of surgery and medicine. ${ }^{35-41}$

Herein, we make a concise review of tissue adhesives with unique chemical properties and their recent applications and developments. Specifically, mussel-inspired dopamine-based tissue adhesives for biomedical application will be the focus of discussion in terms of their key properties, mechanism and applications. Other types of tissue adhesives will also be explained and compared with the dopamine-based adhesives.

\section{Mechanisms of tissue adhesives}

\subsection{Ideal properties of a tissue adhesive}

Wound healing is a complex and dynamic process of replacing devitalized and dead cellular structures and tissue layers that normally occurs through the scar tissue formation. Tissue adhesives as one of the promising alternatives in primary wound closure affect wound healing procedure from different aspects, ${ }^{42-46}$ although existing tissue adhesives still have certain limitations in their performance that call for further efforts in research and development. For example, there have been reports indicated that tissue adhesives may lead to inappropriate wound edges apposition and hence delay wound healing procedure; and the breaking strength of wounds closed with tissue adhesives may be remarkably low in comparison with taped wounds. ${ }^{47-49}$ In addition, the tissue adhesives may potentially contribute to the infection in contaminated wounds. For example, in Staphylococcus aureus contaminated wounds, the occurrence rate of gross infection was considerably greater in wounds closed with tissue adhesives in compared to wounds closed with tapes. ${ }^{\mathbf{5 0}}$

There are several ideal properties that would generally be expected for a tissue adhesive developed for wound healing: both the tissue adhesive and its degradation products should be compatible with tissues. Therefore, researchers are confronted with the challenge of developing a reactive monomer that provides rapid polymerization, and using biocompatible initiators. The tissue adhesive should adhere to a wet or moisture surface at approximately body temperature. Furthermore, surgical tissue adhesives should provide proper applying time, biodegradability, strong and flexible bonding, spreading capacity and wettability. ${ }^{51}$

Mussel-inspired dopamine-based tissue adhesives were well known for their remarkable water resistance characteristic in addition to its biocompatibility, which makes them a potential ideal tissue adhesive for both inside and cutaneous wound closure and healing. Dopamine as a small molecule compound containing catechol and amine groups confers functionality of highly adhesion in wet conditions such as human body. ${ }^{52}$

\subsection{Mussel inspired biomimetic tissue adhesives}

Mussel adhesive proteins secreted from marine mussels' feet are responsible for their ability to from strong adhesive interaction with different type of substrates in a wet condition. Studies suggest that these proteins are highly rich in lysine and modified amino acid, 3,4-dihydroxyphenyl-L-alanine (DOPA) which contain catechol functional group and that these components may confer the observed adhesive properties. ${ }^{\mathbf{5 2 , 5 3}}$ Although the detailed and accurate crosslinking mechanism is still unknown, it is suggested that oxidation of DOPA by means of metal ions or enzymes forms quinone which is essential for the adhesion to organic/inorganic surfaces or tissues. ${ }^{54}$ Thus, during maturation in adhesive plaque of mussel, the quinone moieties crosslink with other quinones using radical mechanism, thiols and/or amines using Michael addition. ${ }^{\mathbf{4 0 5 5}}$ It is believed that the strong adhesion of mussels to a surface is based upon the strong covalently and noncovalently bonding with surfaces procreated by DOPA's phenolic hydroxyl or quinone groups. Moreover, lysine plays a significant role in crosslinking that resulted in solidification of secreted liquid protein adhesive. Enhancing the catechol content provides too much crosslinking, extra cohesion in system and hence lower surface attachment. ${ }^{56,57}$ The abundant amino and hydroxyl groups in these catechol and amine groups also allow them to be easily conjugated to a wide range of material surfaces.

Recently, dopamine has piqued substantial interest in chemistry and biomedical research owing to a similar chemical structure to that of combined lysine and DOPA, as well as its adhesive potential. ${ }^{57}$ Dopamine constitutes one of the most abundant catecholamines in the human body that has long been investigated as a hormone or neurotransmitter, and abnormal levels have been shown to contribute to serious brainrelated disorders. Under alkaline conditions, the catechol functional group oxidizes to quinone in order to provide condition for dopamine self-polymerizing and forming a thin film on support surfaces via covalent bonding and other strong intermolecular interactions like hydrogen bonding, metal chelation, and $\pi-\pi$ interactions. ${ }^{58}$ The chemical structures of DOPA and dopamine are shown schematically in Fig. 2.

Adhesive proteins have been extracted from mussels and examined for biomedical applications. There have been extensive studies on materials surface functionalization using DOPA as a spacer to enhance their adhesion capacity. In recent works, both dopamine and DOPA have been added to different type of polymeric structures to develop highly functional 


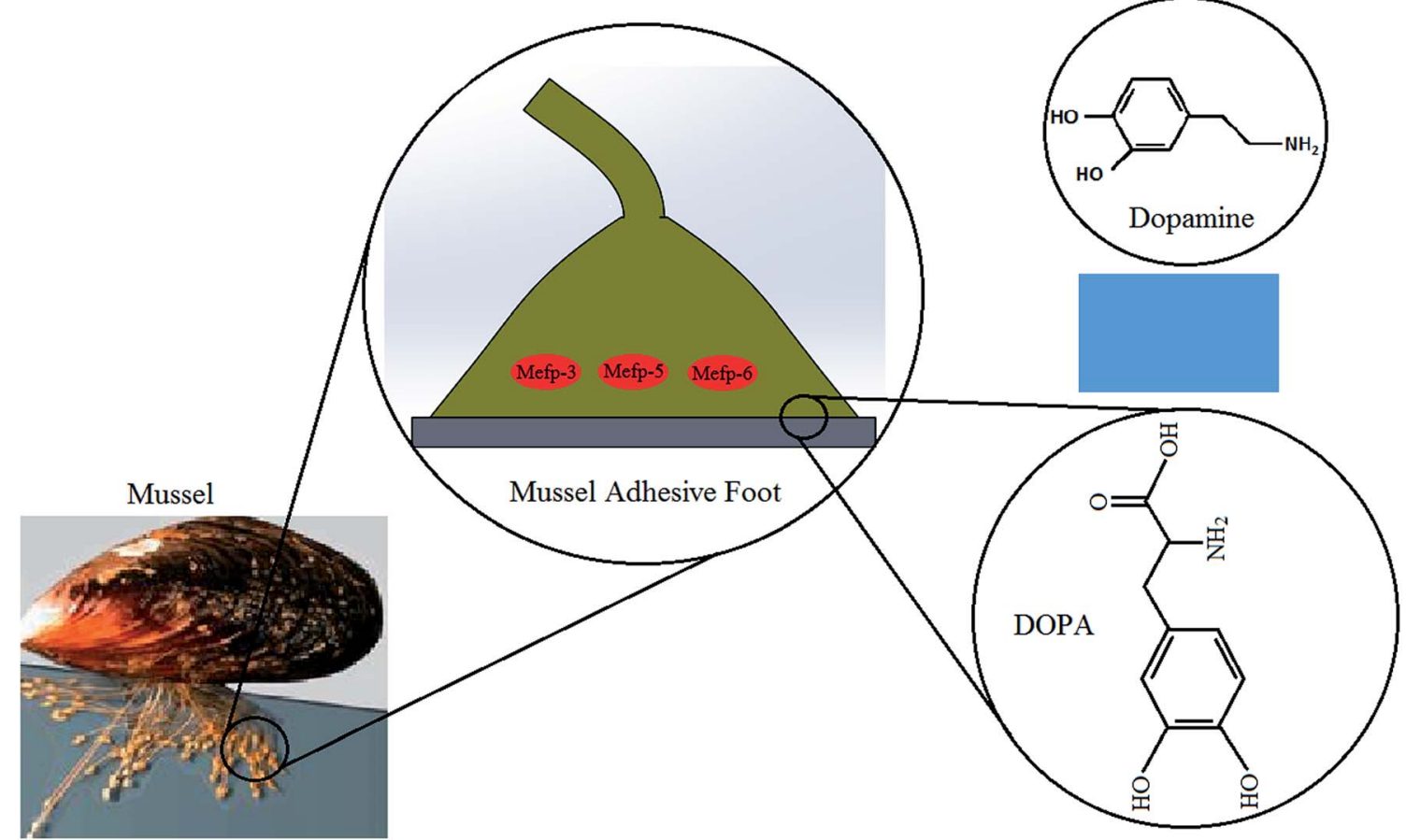

Fig. 2 Molecular structures of DOPA and dopamine (the mussel picture ${ }^{63}$ reproduced by permission of the Company of Biologists).

biocompatible tissue adhesives suitable for wet and dry conditions. ${ }^{53,58-60}$ Many researchers also explore the applications of dopamine-based tissue adhesives by mimicking mussel DOPA. ${ }^{40,61,62}$

\section{Formats of tissue adhesives}

\subsection{Tissue glues}

Existing tissue glues on market generally are not perfect in terms of biocompatibility and biostability. For instance, cyanoacrylate-based adhesives not only cause compatibility issues by releasing formaldehyde degradation products, but also have low mechanical properties; hence they produce inflammation and tissue necrosis. Fibrin glues may show poor adhesion and generate a viral infection risk. In addition, tissue glues are more costly in compared to traditional wound closure methods such as suturing.

As an effort to improve the performance of tissue adhesives, nature inspired tissue adhesives have been developed to provide highly biocompatible yet affordable products. ${ }^{64-67}$ Among them, DOPA or dopamine compounds have attracted extensive attention toward the fabrication of highly efficient tissue adhesives.

Vollenweider et al. used DOPA as a crosslinking precursor to enhance the wet surface adhesion of an adhesive composed of polyethylene glycol (PEG) and polycaprolactone (PCL). ${ }^{68}$ Shao and Stewart fabricated medical glue suitable for open surgery wound closure with a composition of dopamide, polyphosphate, poly-aminated gelatin, and divalent cations. ${ }^{69}$ Choi et al. produced recombinant mussel adhesive protein fp-5 (MAP fp-5) to develop bulk tissue adhesive via various vector systems and cDNA codon optimization. ${ }^{66}$
Recently, a mussel-inspired nano-composite tissue adhesive for sternal bone closure under wet environment was developed to consist of hyper-branched poly( $\beta$-amino ester) (PAE)/(polydopamine-co-acrylate) (PDA) and hydroxyapatite nanoparticles with increased wet adhesion and reinforced structure. ${ }^{70}$ Another research team developed an adhesive precursor composed of hyper-branched PAE polymer modified by catechol groups to improve commercial cyanoacrylate and fibrin bioglues. ${ }^{71}$ Zhou et al. investigated adhesion characteristics of amino acid-based poly(ester urea) (PEU) copolymers functionalized by catechols, demonstrating that the chemical and physical properties of the copolymer can be controlled via varying the amount of diols and amino acids, which is an desirable property for bioglue. ${ }^{72}$ Ahn et al. synthetized low molecular weight catecholic zwitterionic adhesive with a simpler structure as compared to mfp- 5 and mfp-3. The adhesive was shown to have higher adhesion energy than mfp-5. ${ }^{73}$ Jeon's team used a dityrosine photo crosslinking technique in recombinant mussel adhesive protein DOPA, in which tyrosine residues crosslink with each other assisting photo-oxidation reaction. ${ }^{49}$ Lee et al. developed a biomimetic dental adhesive composed of poly dopamine methacrylatemethacryloyl chloride (PDMA-MEA) as the backbone and catechol and methoxyethyl groups in the side chains. The adhesives were demonstrated to have high adhesion, shear strength and biocompatibility due to catechol crosslinking. ${ }^{74}$

Ji et al. created bioadhesives comprised of poly(ethylene oxide) (PEO) and 1,8-octanediol, which have varying capacity from water-soluble to low in swollen by regulating the ratio of PEO to 1,8 -octanediol. ${ }^{75}$ Hong et al. showed two metallobioadhesives, gelatin/Fe and dopamine-modified gelatin $(\mathrm{GelDA} / \mathrm{Fe})$ for wound closure. Based on the characterizations, 
Gel/Fe showed a greater strength whilst GelDA/Fe performed highly acceptable sealing property and seemed to be a better option as tissue glue. Both adhesives were found to have the capacity to induce cells adhesion and growth on the materials surfaces. $^{76}$

Dopamine-based synthetic adhesives have been demonstrated to provide strong bonding between tissue and glue, appropriate strength in mechanical interlocking of glue with underlying tissue and improved cosmetic outcomes. On the other hand, they show slow degradation within 7 to 10 days and released histotoxic components from degraded polymer. These drawbacks can be usually improved by introducing hydrophilic polymers with biocompatible degradation products such as lactic acid and PEG. However, irradiation for polymerization which damages cells and tissue integrated with glue, weak internal strength and cohesion, large swelling in wet condition and inducing foreign body reaction still limited their medical applications. ${ }^{62,77}$

\subsection{Hydrogel adhesives}

Hydrogels exhibit capabilities that make them promising alternatives to be used as tissue adhesives in biomedical applications, including wound closure and injectable drug release systems. The hydrophilic and crosslinked structure endows hydrogels soft tissue like characteristics. In addition, hydrogels have high permeability to oxygen, nutrients, and other water-soluble metabolites. ${ }^{78,79}$ Therefore, hydrogels are becoming a popular format of tissue adhesives. Biomimetic, mussel-inspired tissue adhesive hydrogels were shown to have good healing capacity in the byssal threads of marine mussels ${ }^{\mathbf{8 0}}$ and promising potential to be used as biomedical adhesives. ${ }^{\mathbf{8 1 - 8 4}}$ There have been different strategies to incorporate the DOPA or dopamine functional groups onto the materials (Fig. 3) and to further endow additional functions to the tissue adhesives, as discussed in this section.

3.2.1 Incorporation of dopamine functional or DOPA. Surface of hydrogels can be modified by dopamine to endow adhesive capacity. Cells growth is a key feature in some surface modifications for tissue engineering application. Mussel inspired adhesive coatings provide a promising approach in this field. The amine functional groups on dopamine enable its conjugation to a variety of surfaces of biomolecules. ${ }^{85}$ An et al. modified adhesion property of substrates surfaces by dopamine conjugated polyaspartamide (PolyAspAm), which is a biocompatible and biodegradable adhesive hydrogel. This adhesive was coated onto several substrates such as metals, oxides, synthetic polymers and ceramics by an immersion method to provide a biocompatible surface for various substrates. ${ }^{\mathbf{8 6}}$

In addition to surface modification for surface bioadhesion, various studies have been focused on conjugation of dopamine or catechol functional groups onto both natural and synthetic polymers. Such modifications not only affect the bioadhesive properties, but also the mechanical strength of the hydrogels. The most frequently used polymers for such purposes are discussed below.
Chitosan is a natural polymer that has been used to develop adhesive hydrogels. Xu et al. reported three chitosan/catechol hydrogels with difference types of catechols, including DOPA, hydrocaffeic acid (HCA), and dopamine to compare their swelling ratio and adhesion to the mucus. Results demonstrated that the swelling ratio and adhesion can be altered by the nature of catechol compounds, with HCA/chitosan hydrogels showed the lowest swelling ratio and highest mucus adhesion. $^{87}$

Gelatin is a popular natural polymer for hydrogel fabrication. The adhesive capacity of an injectable gelatin hydrogel was improved by using DOPA. Upon addition of $\mathrm{Fe}^{3+}$ ions, the gelatin modified by DOPA can be converted into an adhesive hydrogel via DOPA- $\mathrm{Fe}^{3+}$ coordination and quinone coupling. After injection to a hemorrhaging site of a rat liver, the adhesive demonstrated good hemostasis by producing adhesive obstacle on site of bleeding. Also this adhesive hydrogel showed appropriate mechanical strength and good biocompatibility to be used in medicine and surgery. ${ }^{88}$ Zhu et al. developed dopamine based tissue adhesive containing gelatin as a backbone, genipin and $\mathrm{FeCl}_{3}$ as the slow and fast crosslinker, respectively. Its effect on mastectomy surgery in a rat model was examined. Acceptable tissue adhesion, mechanical strength, biocompatibility and minimized formation of seroma were reported. ${ }^{89}$ Dual crosslinking method was used to prepare dopamine based tissue adhesive with gelatin polymer backbone. Fan et al. created a gelatin adhesive which is capable to be used in both external (skin) and internal (cartilage) wound closure applications. The results showed high wet adhesion, promising biocompatibility and biodegradability. ${ }^{90}$

Another research group designed hyaluronic acid (HA) sticky hydrogels modified by a catecholamine cross linker for cell therapy. Catecholamine consisting of catechol group could improve HA functionality and cell adhesion. The catechol group oxidized to $o$-quinone was operated in alkaline $\mathrm{pH}(>7.5)$ condition to crosslink chemically catechol-catechol compositions, then the catechol moieties were conjugated to the HA hydrogel. The modified HA gel structure exhibited higher tissue adhesion and biocompatibility in comparison with HA hydrogel.91

Polyethylene glycol (PEG) or polyethylene oxide (PEO) is a very popular synthetic polymer for developing adhesive hydrogels. Brubaker et al. examined the use of PEG hydrogel with catechol functional group on extrahepatic islet transplantation which had potential application for diabetic patients. Direct immobilization of islets onto intra-abdominal tissue surface through the hydrogel adhesive in mice was achieved. ${ }^{92}$ Barret et al. developed mussel protein based amphiphilic poly(propylene oxide) (PPO)-poly(ethylene oxide) (PEO) polymer hydrogels that can be formed in situ for surgical wound treatment. The hydrogel demonstrated stresses of 3.4-4.3 MPa and $90 \%$ strain without any rupture and damage. It can tolerate $90 \%$ compression at body temperature in an equilibrium swollen phase. Higher roughness and negative swelling ratio can be achieved by modification of PEO using PPO and catechol functional groups. ${ }^{93}$ A fast curable hydrogel made of DOPA, dextran and three-arm PEG was prepared by linking DOPA to 


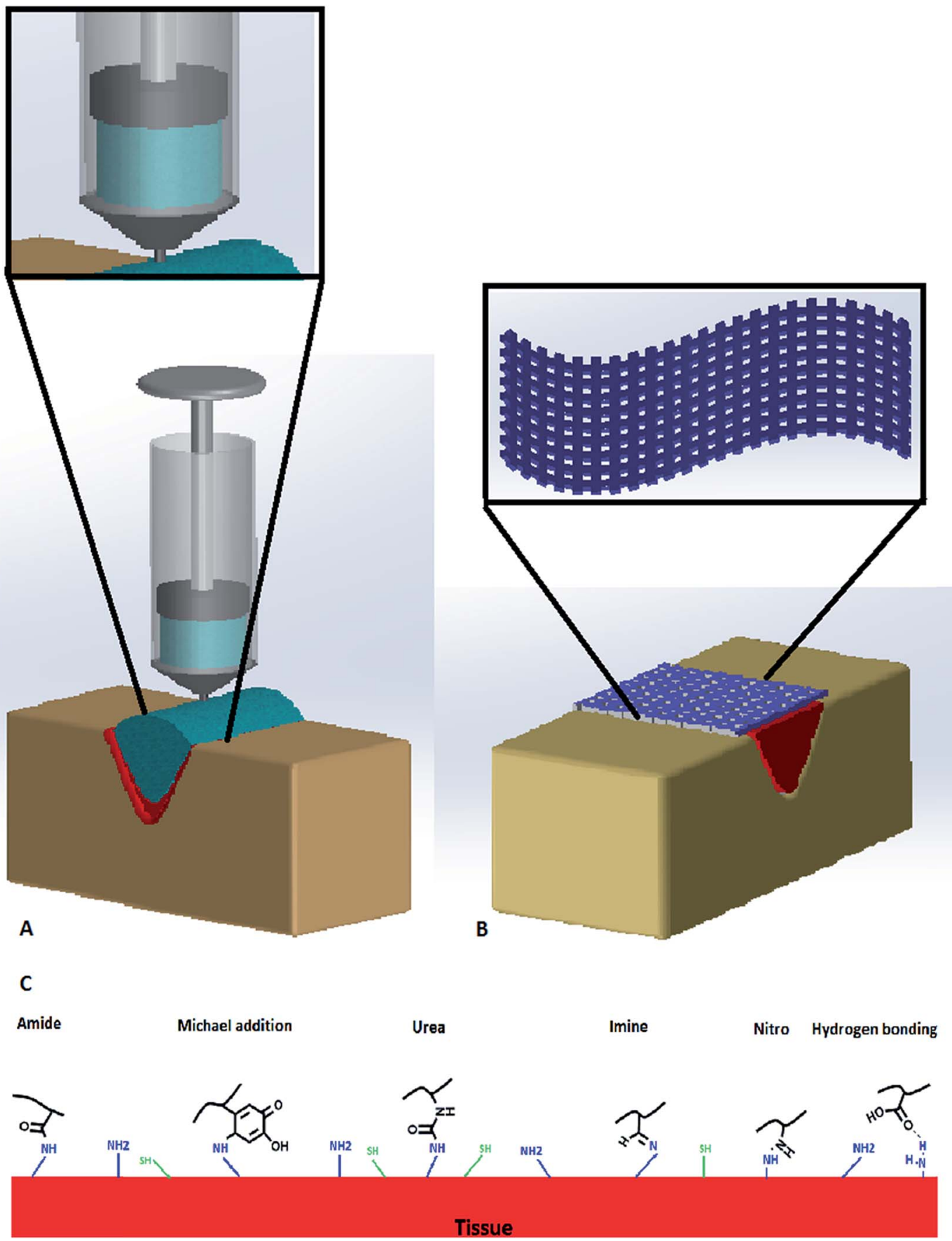

Fig. 3 (A) Dopamine-based injectable adhesive hydrogels in wound closure, (B) dopamine-based nanofibrous adhesive mat in wound closure, and (C) dopamine functional groups reaction with tissue.

PEG-TA (tetraacrylate) by Michael reaction between acrylate double bonds and amine groups. Usage of PEG-DOPA increased the adhesion strength of the dextran hydrogels from 2.7 to $4.0 \mathrm{MPa} .{ }^{94}$ Also, Liu et al. modified and improved adhesion and bio interaction properties of PEG based tissue adhesive by applying dopamine, catechol groups and nanoparticles. They indicated that the nanocomposite demonstrated enhanced mechanical properties, rate of curation and bioadhesion because of strong conjugation between LAPONITE® and dopamine. ${ }^{95}$
Skelton et al. developed polyacrylamide (PAAm)/dopamine methacrylate (DMA) hydrogels incorporated with nano silicate and LAPONITE®. Photo polymerization enabled covalent linkage of catechol with hydrogel of PAAm containing nano silicate. The final hydrogels showed improved rigidity and viscosity that affect the rheology involving storage and loss moduli. ${ }^{96}$ Jenkins et al. carried out a study to characterize the effect of molecular weight of biomimetic proteins on adhesion of polymer. It was suggested that a higher adhesion stems from higher molecular weight. Though achieving high 
adhesion strength ranging in $\mathrm{MPa}$ is usually difficult for dopamine-based hydrogels due to their high water content. ${ }^{97}$

Zhou et al. assessed adhesion characteristics of mussel inspired dopamine based PEU gel. The findings confirmed phenylalanine, tyrosine, and catechol functional groups affect adhesion through contact angle measurements and JohnsonKendall-Roberts (JKR) method: cross-linking oxidation of catechol remarkably increased lap-shear adhesion strength and catechol modified PEU showed better adhesive strength compared with fibrin glue on porcine skin. ${ }^{72}$

An adhesive hydrogel used for peripheral nerve regeneration contained of chitosan and $\varepsilon$-polylysine (PL) was prepared by Zhou et al. that was able to form in situ and simulate chemical structure of natural epineurium matrices which increases its nerves biocompatibility. Maleimide and thiol functional groups were allowed to react with each other by Michael-type addition, to form a cross linker to speed up curing time and eliminate further damages to nerves. Rapid gelation in $10 \mathrm{~s}$ prompted sealing of transected nerves. The morphology of the repaired nerve of sealed with the adhesive hydrogel after 8 weeks was similar to that of the normal nerve. Its functionality was evaluated in vivo on rat models, hence demonstrated potential application for nerve surgery. ${ }^{\mathbf{9 8}}$
There have been plenty of reports on DOPA or dopamine incorporated hydrogels that combine both natural and synthetic polymers. Burke and colleagues developed a PEG/silk fibroin adhesive hydrogel in which dopamine was chemically coupled to silk fibroin enriched with carboxyl groups. PEG controlled the hydrophobicity of the fibroin backbone and the gel solubility that was incorporated. ${ }^{99} \mathrm{Li}$ et al. focused on dopamine-modified poly(ethylene glycol) (PEGDM) adhesives incorporating gelatin microgel in order to increase adhesion and bioactivity. They compared the PEGDM hydrogel which combined with gelatin microgel properties with gelatin microgel. PEGDM containing gelatin microgel adhesive properties improved from 1.5 to 2 fold, promoted attachment of fibroblasts and cell viability regarding it implanted in rat, and improved rheology parameters such as enhanced loss moduli. The loss modulus and storage modulus that represent adhesive elasticity enhanced by gelatin microgel content increasing which indicated reversible physical bonding. ${ }^{\mathbf{1 0 0}}$ A citrate based tissue adhesive hydrogel (POECd) was fabricated using melt polycondensation of four agents of 1,8-octanediol, PEO, citric acid (CA) and dopamine. Effects of PEO on swelling, mechanical and biocompatibility of adhesive hydrogel was also examined. The adhesive strength and viability demonstrated that the lap shear adhesion strength, in the range of $21.7-33.7 \mathrm{kPa}$ was higher than commercial fibrin glue lap shear adhesion strength which

Table 1 Polymeric hydrogels incorporated with DOPA or dopamine functional group

\begin{tabular}{ll}
\hline Polymer & Methods of incorporation of DOPA/dopamine \\
\hline Chitosan & $\begin{array}{l}\text { Mixed hydrogels of chitosan and a catechol- } \\
\text { containing compound: DOPA, hydrocaffeic acid } \\
\text { (HCA), or dopamine } \\
\text { Catechol groups conjugated onto PL molecules in } \\
\text { a nerve adhesive hydrogel composed of chitosan } \\
\text { and PL }\end{array}$ \\
Chitosan \& E-polylysine & $\begin{array}{l}\text { Polycondensation of citric acid (CA), PEG and } \\
\text { catechol-containing compound } \\
\text { Adhesive hydrogel containing gelatin modified by } \\
\text { Citrate polymer and } \\
\text { derivatives }\end{array}$ \\
Gelatin & $\begin{array}{l}\text { DOPA via DOPA-Fe } \\
\text { coupling }\end{array}$
\end{tabular}

Hyaluronic acid (HA)

Polyethylene glycol (PEG) or polyethylene oxide (PEO)

PEG (three-arm) \& dextran

Polyacrylamide (PAAm)

Polyaspartamide

(PolyAspAm)

Poly(ester urea) (PEU)

Silk fibroin
HA hydrogels functionalized by catechol via oxidative crosslinking

PEG, PEO or their derivative poly (ethylene glycol) dimethacrylate (PEGDMA) conjugated with catechol groups

DOPA linked to PEG-TA (tetraacrylate) by Michael reaction between acrylate double bonds and amine groups

Copolymerization of DMA (dopamine methacrylamide) into PAAm hydrogels Material surface modified by being immersed in a coating solution containing PolyAspAm conjugated with dopamine

Incorporation with phenylalanine, tyrosine, and catechol groups

Dopamine chemically coupled to silk fibroin enriched with carboxyl groups
Property features

Ref.

Mucoadhesion of chitosan hydrogels

87

increased by the incorporation of catechol-containing compounds

Incorporation of catechol groups was

shown to enhance both mechanical and

nerve adhesion properties of the

hydrogels

Higher adhesion strength and lower

toxicity compared to fibrin glue

Good hemostasis, appropriate

mechanical strength and good

biocompatibility for applications in surgery

Modified HA gels with higher tissue adhesion and biocompatibility

Enhanced mechanical properties and

bioadhesion, negative swelling ratio,

promoted attachment and viability of cells

Enhanced adhesion strength

101-103

$88-90$

91

92, 93, 95 and 100

94

Improved mechanical and rheological properties

Bioadhesive surface achieved on various

86 substrates including metals, oxides, synthetic polymers and ceramics

Increased adhesion

$\beta$-Sheet structures of silk fibroin not affected 
was in the range of 9-15 $\mathrm{kPa}$ and lower toxicity compared to fibrin glue. ${ }^{75}$ Citrate polymer and its derivatives showed intrinsic antimicrobial behavior. Guo et al. synthetized citrate based antimicrobial bioadhesives composed of citric acid and catechol functional group. ${ }^{\mathbf{1 0 1}}$ Such adhesives demonstrated 13 times higher adhesion strength as compared to fibrin glue. They also showed antimicrobial properties due to dual crosslinking procedures including click chemistry crosslinking and DOPA crosslinking. ${ }^{102}$ Methods of incorporation of DOPA or dopamine functional group into mussel inspired tissue adhesives and their property features are summarized in Table 1.

3.2.2 Self-healing. Self-healing hydrogel adhesives can provide remarkable advantages in wound closure applications to remedy transit damage caused by rough tactility (Table 2). For instance, if a self-healing hydrogel adhesive is used in drug delivery systems, hydrogels damaged by external forces can restore to an integral piece under physiological temperature or $\mathrm{pH}$, which can prevent the full release of loaded drugs and increase the effectiveness of drug delivery system. ${ }^{81}$ The risk of bare implant surfaces contacting with body fluids will be decreased by coating implant using self-healing tissue adhesives, because the damages generated on implants coating which may occur in surgery, could be autonomously repaired. ${ }^{\mathbf{1 0 4}}$ Ahn et al. suggested a metal-free strategy to create the selfhealing adhesive polymers under wet condition, via hydrogen bonding between catechol moieties. They employed semi rigid polyacrylate and rigid polymethacrylate to synthetize polymer consisting of pendant catechol protected by silyl groups. Due to removal of silyl groups to reveal catechol functional groups, the adhesive hydrogels provided self-healing behavior in acidic solution, although they could not observe any self-healing behavior from hydrogels in neutral to alkaline solutions, in which the catechol groups were protected. These results demonstrate promising self-healing properties of mussel byssus and also inspired the development of new self-repair adhesive hydrogels for various wound treatments. ${ }^{\mathbf{1 0 5}}$

Some studies not only concentrated on wet adhesion of mussel inspired tissue adhesive hydrogels, but also considered their self-healing properties. The metals and mussel foot proteins' coordinative bonding which is able to be reversed, attribute mussel byssus self-healing properties. This metal induced protein complexation mechanism motivated creation of self-repair polymer hydrogels with controlled features. In this regard, DOPA- $\mathrm{Fe}^{3+}$ noncovalently cross-linked hydrogels were extensively studied. Holten-Andersen et al. synthetized a PEG based adhesive hydrogel containing catechol groups in which the mechanical properties and self-healing behavior could be controlled by $\mathrm{pH}$ changes. This study exhibited that the catechol groups can produce a mono-complex with ferric ions in $\mathrm{pH}<5.6$, bis-complex in $\mathrm{pH}$ between 5.6 and 9.1 and tris-complex in $\mathrm{pH}$ greater than 9.1. Hydrogels with covalent cross-linking at high $\mathrm{pH}$ resulted in catechol- $\mathrm{Fe}^{3+}$ cross-linked hydrogels, show elastic moduli. The hydrogels cross-linked by tris-catechol-Fe ${ }^{3+}$ were shown to rapidly restore to its stiffness and cohesiveness,

Table 2 Self-healing, pH sensitive $\&$ temperature sensitive mussel inspired hydrogel tissue adhesives

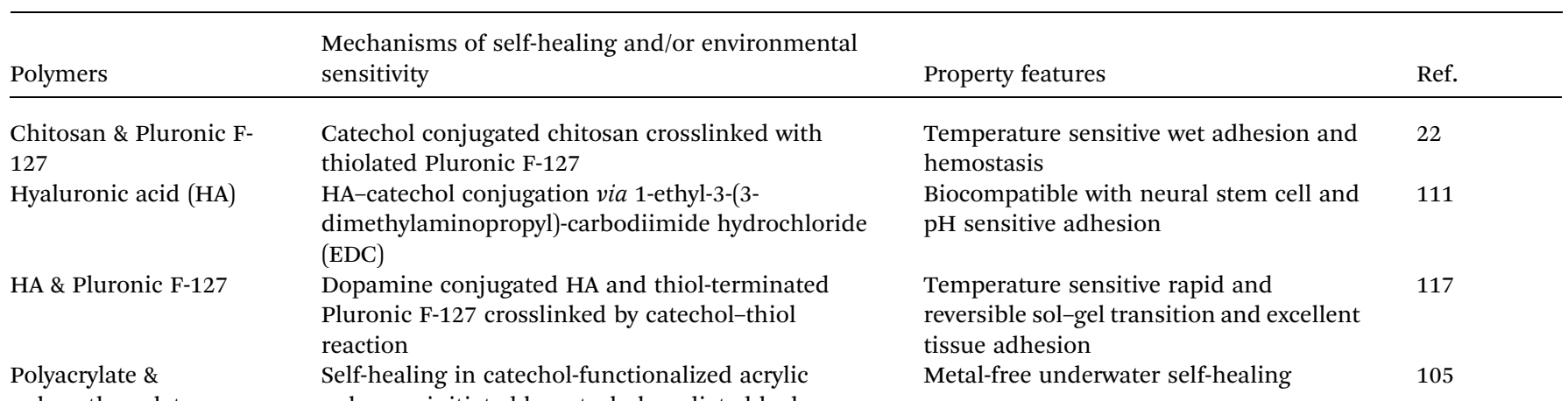

polymethacrylate

Polyallylamine

Poly(ethylene glycol) (PEG)

Poly(dopamine methacrylate- $\mathrm{Co}-\mathrm{N}$ isopropylacrylamide) Polyurethanes (PU) polymers initiated by catechol-mediated hydrogen bonding

DOPA/iron coordination in response to $\mathrm{pH}$ in DOPA-functionalized polyallylamine pH-induced metal-ligand (catechol-Fe ${ }^{3+}$ ) crosslinking in DOPA-modified PEG

Phenyl borate-catechol complexation in hydrogels containing dopamine functionalized 4-armed PEG and phenylboronic acid modified 4-armed PEG Four-armed PEG end-capped with dopamine Hyperbranched PEG incorporated with catechol and photocrosslinkable methacrylate groups Boronate ester linkages provide self-healing properties at alkaline condition

PU hydrogels containing hexamethylene diisocyanate as hard segment, PEG as soft segment, and lysine-dopamine as side chain
High mechanical strength and fast selfrepair behavior in alkaline condition Crosslinked networks with elastic moduli approaching covalently cross-linked gels and with self-healing

Self-healing hydrogels with $\mathrm{pH}$, glucose, and dopamine triple responsiveness and biocompatibility

pH dependent adhesive performance Provide on-demand photocuring and strong adhesion

Rapid self-repair adhesive polymer and multiple stimuli-responsive behavior

Rapid pH sensitive gelation through Fecatechol complexes 
that may be ascribed to the broken catechol- $\mathrm{Fe}^{3+}$ crosslinks rehabilitation. ${ }^{\mathbf{1 0 6}} \mathrm{A}$ pH responsive hydrogel was developed through bonding DOPA to an intrinsic $\mathrm{pH}$ responsive polymer which was functionalized by amine groups. The hydrogels that reacted with ferric ions, demonstrated high mechanical strength and fast self-repair behavior in alkaline condition. ${ }^{\mathbf{1 0 7}}$

Another self-repair metal-catechol based adhesive hydrogel was prepared utilizing dynamically reversible $\mathrm{B}^{3+}$-catechol complexation. Catechol group is able to create stable covalent conjugation with boronic acid structure at neutral and alkaline condition and dissociate in an acidic pH. He et al. fabricated a self-repair hydrogel using the complexation of catechol crosslinking, PEG derivatives and 1,3-benzenediboronic acid. ${ }^{\mathbf{1 0 8}}$ The boronate ester linkages could provide self-healing behavior and completely mechanical deformation recovery at alkaline condition in hydrogels. Poly(dopamine methacrylate- $\mathrm{Co}-\mathrm{N}$-isopropylacrylamide) and boric acid $\left(\mathrm{H}_{3} \mathrm{BO}_{3}\right)$ were used to develop a rapid self-repair adhesive polymer with multiple stimuliresponsive behavior, which approves strong $\mathrm{pH}$ and catechol concentration dependent functionality of $\mathrm{B}^{3+}$-catechol coordinated in the hydrogels. ${ }^{109}$ A multi-sensitive catechol based adhesive hydrogel was fabricated by Shan et al. via catechol and phenylborate incorporation. Such adhesives demonstrated selfhealing property and responsiveness to $\mathrm{pH}$, glucose and dopamine. Cytocompatibility and high adhesion strength were reported, indicating its potential application in drug delivery systems and tissue regeneration. ${ }^{\mathbf{1 1 0}}$

3.2.3 pH sensitive. Mehdizadeh et al. synthetized citrate and DOPA based hydrogel including citric acid, PEG and dopamine or L-DOPA, resulting in hydrogels with high adhesive capacity in highly wet conditions as well as efficient mechanical properties, controlled biodegradation rate and good biocompatibility for wound sealing application. DOPA may boost crosslinking reactions via catechol hydroxyl side chains oxidation in alkaline environment, afterward prompting intermolecular crosslinking as well as bulk cohesive strength. In addition, oxidized DOPA provide the strong wet adhesion to biological surfaces, as these surfaces bond covalently with available nucleophilic groups such as- $\mathrm{NH}_{2},-\mathrm{SH},-\mathrm{OH}$, and-COOH. ${ }^{62}$ Beside, in neural disease treatment and tissue engineering, 3D hydrogel scaffolds consisted of HA conjugated with catechol groups were fabricated for encapsulating neural stem cells. This biomimetic construct demonstrated $\mathrm{pH}$ sensitive adhesion and cohesion considering wound $\mathrm{pH}$ condition and showed potential to be used as tissue adhesives. ${ }^{111}$ Sun et al. created dopamine and lysine groups based polyurethanes (PU) injectable bioadhesive using step growth polymerization. The $\mathrm{pH}$ changes accelerated polymer structure gelation through $\mathrm{Fe}$-catechol complexes transmutation. ${ }^{\mathbf{1 1 2}}$

There have been research on the influence of environmental pH on curing rate and tissue adhesion of dopamine based PEG hydrogels. It was suggested that an acidic condition decreases curing rate and adhesive strength, while an alkaline environment lessen tissue adhesion due to reduced concentration of catechol. Formulated hydrogel showed good curing rate, improved mechanical properties and adhesion at $\mathrm{pH} 7.4 .{ }^{113}$ Nitro-group functionalized dopamine-based four-arm PEG was analyzed to achieve optimum adhesion, strength and rate of curing in presence of different concentration of $\mathrm{NaIO}_{4}$ and $\mathrm{pH}$. Nitrodopamine-based PEG hydrogel had much more constant adhesive strength in $\mathrm{pH}$ ranges from 5.7 to 8, while dopaminebased ones had higher adhesive strength in neutral (body normal $\mathrm{pH}$ ) and alkaline $\mathrm{pH}$ ranges. ${ }^{\mathbf{1 1 4}} \mathrm{Kim}$ et al. fabricated an adhesive hydrogel by using DOPA-comprising recombinant mussel adhesive proteins to provide strong adhesion strength, flexibility, appropriate viscoelasticity and self-healing properties. The adhesive strength of the $\mathrm{Fe}^{3+}$-mediated noncovalent crosslinked hydrogel was measured to be around $130 \mathrm{kPa}$ in alkaline $\mathrm{pH}$ environment, whilst the quinone-mediated covalent hydrogel adhesive strength improved with curing time up to close $200 \mathrm{kPa}$, after $30 \mathrm{~min} \mathrm{NaIO}_{4}$ treatment. The recombinant mussel adhesive proteins produced from traditional biosynthesis system that do not have DOPA, thus the subsequent procedure of tyrosine residues transformation into DOPA molecules suffers from a low modification yield. ${ }^{\mathbf{1 1 5}}$ To find a solution for this problem, an in vivo residue-specific incorporation strategy was adopted by Yang et al. to develop engineered mussel adhesive proteins in Escherichia coli with a very high amount of DOPA which was $16.5 \mathrm{~mol} \%$ in comparison with natural ones (20-25 mol\%). This protein exhibited enhanced wet adhesion capacity as demonstrated via surface forces apparatus (SFA) measurements and quartz crystal microbalance (QCM) analysis, showing potential application as bioadhesives in biomedicine. ${ }^{\mathbf{1 1 6}}$

3.2.4 Temperature sensitive. An adhesive hydrogels were designed by crosslinking hyaluronic acid and thiol-terminated Pluronic F-127 via Michael type catechol-thiol addition by Park and colleagues in which the structures were functionalized by catechol groups. ${ }^{117}$ As a result, a new mussel inspired temperature sensitive wet adhesive was fabricated. Chitosan/ Pluronic F-127 copolymer hydrogels with catechol and thiol groups were fabricated through covalent bonding of catechol conjugated with chitosan and thiol of Pluronic to provide wet bioadhesion and hemostasis. ${ }^{22}$ When the sol injection was done in vivo at room temperature, the precursor solution was able to form a strong and robust hydrogel rapidly at body temperature. Universal testing machine (UTM) was used to evaluate adhesion properties, and the detachment stress value of HA/Pluronic hydrogel and chitosan/Pluronic hydrogel were measured as about $7.18 \mathrm{kPa}$ and $15.0 \mathrm{kPa}$, respectively. ${ }^{22,117}$ Dual PEG/ catechol conjugated gel polymers were investigated by Zhang et al. They analyzed composition and swelling ratio by comparing proportion of acrylates to methacrylates and PEG to 2-hydroxyethyl acrylamide (HEAA). They developed hyper branched thermos-responsive hydrogels combining PEG and catechol through reversible addition fragmentation chain transfer polymerization (RAFT) tested on bovine heart tissue for potentially using in skin and cardiac tissue adhesive applications $^{118}$ (Table 2).

3.2.5 Drug delivery systems. A bioinspired hydrogel with a hydrophobic polyanhydride backbone grafted with DOPA side chains was developed for potential tissue adhesives in oral drug delivery. The structure represented better fracture strength and tensile strength in compare to polycarbophil, a common 
commercial adhesive in oral drug delivery system (DDS). ${ }^{\mathbf{1 1 9}}$ Alginate/catechol bioadhesive hydrogels were developed to provide durable adhesion to intact endothelium under blood flow and to deliver a steroid drug that stabilizes rupture-prone plaques. This adhesive hydrogel was implanted in the blood vessels in a mouse with atherosclerosis to show potential application in biomedicine. ${ }^{\mathbf{1 2 0}}$ Fullenkamp et al. devised silverreleasing antibacterial adhesive hydrogels and coatings from redox reactions between soluble $\mathrm{Ag}$ (I) and catechol-terminated PEG (cPEG). A bulk hydrogel is produced by blending cPEG and $\mathrm{AgNO}_{3}$ solution. The reduction of $\mathrm{Ag}(\mathrm{I})$ to $\mathrm{Ag}(0)$ was accompanied by catechol functional groups oxidative polymerization in order to form hydrogels, which resulted in an adhesive hydrogel with entrapped Ag nanoparticles. A sustained release of silver nanoparticles from the Ag-cPEG gel was monitored in PBS solution. This adhesive hydrogel coating showed excellent antifouling functionality toward mammalian cell and bacterial attachment. ${ }^{121}$ Polysuccinimide (PSI) is a biodegradable and biocompatible polypeptide in human body. PSI has a potential to be grafted to both hydrophilic and hydrophobic elements in the presence of amine functional groups through its penta ring chemical structure. The product of this chemical open ring reaction is polyaspartamide that is capable to be utilized in nanoparticles for drug delivery systems. Lim et al. took advantages of this biopolymer to fabricate a wet adhesive based on DOPA-grafted PSI with capacities of wound closure and drug release. In vitro and in vivo results confirmed well adhesion and faster healing in comparison with suturing. ${ }^{122}$ Gong et al. also studied PSI as one of $\operatorname{poly}(\alpha, \beta-$ aspartic acid) derivatives conjugation with dopamine in order to create adhesive with drug release feature. $\mathrm{FeCl}_{3}$ was added to coordinate crosslinking between $\mathrm{Fe}^{3+}$ and catechol and enhance cell adhesion. It demonstrated slow and continuous drug release within 4 weeks. ${ }^{123}$

3.2.6 Enzymatically degradable. Considerable effort was dedicated to enhance adhesion strength of hydrogel adhesives. Teixeira et al. investigated hydrogels based on enzyme-mediated crosslinking. ${ }^{124}$ An enzyme-degradable adhesive hydrogel was created by incorporating minimal elastase substrate peptide AlaAla into a branched PEG macro monomer network. It can degrade via neutrophil elastase expression and neutrophils secretion in wounds and inflamed tissues and it demonstrated adhesive strength of $30.4 \pm 3.39 \mathrm{kPa} .{ }^{125}$

Despite of improved biocompatibility and biodegradability of tissue adhesives with hydrogel based structures; they usually demonstrate poor mechanical properties, particularly at loaded parts of the body such as knee meniscus and heart valves. Dopamine based structures provide wet adhesion and strong cohesion between adhesive hydrogel and tissue, thus the robust mechanical strength remains the main concern for researchers. Toxic oxidizing agents like periodate and iron ion utilizing and enhanced degradation time due to strong crosslinking are other disadvantages of hydrogel adhesives. ${ }^{\mathbf{8 8 2 6}}$ Nevertheless, DOPA functionalized hydrogel polymers show great performance to be used as tissue adhesives, though no clinical studies have been done to date.

\subsection{Micro/nano fibrous films, sheets \& patches}

To explore the application of tissue adhesives in care for different wounds such as surgical, burn wounds and diabetic ulcers, improve mechanical properties and to increase strengths of tissue adhesives, there have been recent studies on the development of tissue adhesives in the form of film, mat or sheet. ${ }^{127,128}$ To date, a few studies concentrate on nature bioinspired particularly dopamine based nanofibrous tissue adhesive sheet or film as advanced materials for wound closure (Fig. 3B).

Lee et al. studied performance and functionalities of mussel and gecko inspired tissue adhesives in wet and dry conditions. They prepared a bio inspired adhesive including of nanostructure polymer pillars which were coated by a polymer that contains mussel derived adhesive proteins. The mussel proteins increased wet adhesion of the nanostructure film for several contact cycles in both dry and wet surfaces, which showed reversible adhesion on different surfaces. ${ }^{58}$ Later, DOPA based metallopolymer film was created containing $\mathrm{Fe}^{3+}$ and $\mathrm{mfp}-1$, in order to improve hardening and adhesion. Although, healing of byssal threads of marine mussels can rare be achieved by artificial materials typically due to lack of dynamic migration of their building blocks or molecular components across the defects to restore bonds after damage and rupture, they concluded that interactions between DOPA and $\mathrm{Fe}^{3+}$ produce opportunity to engineer self-healing and strong adhesive polymer. ${ }^{129}$ A novel triazine based adhesive was synthetized as primers for sealing of bone fractures in surgeries. The study assessed various phenol functional groups as primers in fabricating fibrous patches. Poly(parahydroxylstyrene) (PHS) and phenolic dopamine was used to form adhesive patches with elevated bioadhesion in wet condition for bone fracture fixations in vitro. ${ }^{19}$ Murphy et al. developed tissue adhesive polymer made of DOPA derivatives and PEG and PCL, providing wet environment friendly biodegradable and biocompatible adhesive film for hernia repair. The film structure was shown to promote rapid tissue ingrowth, to decrease inflammation and enhance lap shear strength as applied on bovine pericardium. ${ }^{\mathbf{1 3 0}}$

Sparks et al. developed an adhesive polymer, in which dopamine acrylamide (DAm) was used to provide adhesion by catechol oxidation. Catechol was incorporated with pentaerythritol triallyl ether (APE) and pentaerythritol tetra(3mercaptopropionate) (PETMP) that provided thiol-ene functional groups. Oxidized catechol was found to result in enhanced adhesion of the coatings to different type of substrates such as glass, aluminum, steel, and marble by varying the DAm amount in the network, as an increase in DAm concentration remarkably decreases the crosslinking density. ${ }^{\mathbf{1 3 1}}$ Kim and coworkers incorporated catechol onto water insoluble chitosan and found that catechol conjugation changes the solubility of chitosan and create an adhesive film. ${ }^{\mathbf{1 3 2}}$ Oh et al. also worked on modification of chitosan to provide films with good wet adhesive capacity by incorporating an oxidant, sodium periodate into mix of chitosan and DOPA. Compared with chitosan film, the resulted film showed 7 fold higher stiffness and lower swelling ratio. ${ }^{133} \mathrm{~A}$ cell adhesive patch was fabricated 
through coating of PDA on poly(lactide-co-caprolactone) (PLCL) fibrous patch by Shin et al. for cardiac cell delivery application. The RGD peptide immobilization was confirmed via surface wettability measurement. Further, they investigated the patch biocompatibility, myoblast cells adhesion, proliferation and differentiation in vitro. ${ }^{\mathbf{1 3 4}}$

Zhang et al. reported synthetizing of PDA and poly( $\mathrm{N}$-isopropylacrylamide) (PNIPAM) adhesive coating and a drug delivery system. The adhesive surface contains liposomes that adsorb hepatocytes, macrophages, and myoblasts to the films. ${ }^{135}$ PDA can be used for metal and metal oxide surfaces modification, and utilized to modify reduced graphene oxide (RGO) through dopamine self-polymerization. Guo et al. fabricated RGO adhesive sheet coated by PDA which was used as a metal ions reducing agent for the cell immobilization and also improvement of surface adhesion. ${ }^{\mathbf{1 3 6}}$ Cheng et al. studied surface modification of graphene oxide (GO) sheet by dopamine in which the morphology was controllable with high capacity of drug loading. They used carbodiimide chemistry technique to graft dopamine onto heparin. Then the dopamine-grafted heparin was adhered to the RGO surface. The developed materials showed good biocompatibility, controlled 2-dimentional morphology and significant drug loading. ${ }^{137}$

Ge et al. used layer by layer (LbL) electrospinning method to fabricate dopamine based nanofibrous structure of PCL/gelatin and increase adipose cell adhesion in bone tissue engineering. The mussel inspired electrospun structure not only improved mechanical strength of the scaffold, but also increased cell attachment through enhancing surface hydrophilicity. ${ }^{\mathbf{1 3 8}}$ Dopamine based coating on HA polymer and multilayer films by using LbL of chitosan and dopamine/HA was done by Neto and coworkers. Films presented cell viability, appropriate biocompatibility and high adhesion mechanics. ${ }^{139}$ Another research group used a solvent free and simple technique to fabricate a thin, flexible and strong polysaccharide film which has potential to be applied in biomedical fields. Catecholamine was used as a crosslinking agent and chitosan as polymer. The tensile strength of the film is approximately $10 \mathrm{MPa}$, which is higher than that of an A4 paper (3.5 MPa). ${ }^{\mathbf{1 4 0}} \mathrm{Jiang}$ et al. devised gelatin/PCL nanofibrous sheet modified with dopamine which was used in surgical wound closure. Dopamine was crosslinked to the gelatin backbone via amino group using photo initiating method. Final adhesive sheet demonstrated efficient suture-less surgical wound sealing in stomach. ${ }^{\mathbf{1 4 1}}$ Poly(L-lysine) (PLL) was incorporated with PDA to prepare an adhesive film as a coating for endothelial cells support improvement. PLL provided highly integration with PDA because of high amount of amine functional groups, subsequently excellent interaction with endothelial cells and enzymatically biodegradation. ${ }^{\mathbf{1 4 2}}$

Recently, Zieger et al. introduced catechol based surface modification with a new catechol derivatives family containing cyclic catechol oligomers to generate homogeneous layer with preserved topography. This dopamine based thin film had potential to prepare multi layered structure through covalent binding of catechol with various substrates such as $\mathrm{Au}, \mathrm{TiO}_{2}$, and $\mathrm{SiO}_{2} \cdot{ }^{143}$ To fix and stabilize bone fracture, Olofsson and colleagues reported fibrous adhesive patch using primers made of dopamine derivatives that consist of different allyl, thiol and methacrylamide groups. A thin primer layer was capable to improve the bone and adhesive interaction. On the other hand, these groups increased shear strength in adhesion. They resulted in non-toxic, remarkable adhesion and $0.3 \mathrm{MPa}$ shear adhesive strength. ${ }^{\mathbf{1 4 4}}$

To maximize the catechol agents' presence, catechol based nanoparticles have been used because of their high surfacevolume ratio. Dopamine oxidative self-polymerization forms the nanoparticles. This procedure is similar to the melanin formation pathway, thus these catechol based nanoparticles have been named as melanin-like nanoparticles. Scognamiglio et al. fabricated an adhesive membrane coated ${ }^{\mathbf{1 4 5}}$ with melaninlike nanoparticles for surgical wound closure. In this study, alginate/hyaluronan membrane was created via molding method $^{\mathbf{1 4 5}}$ and coated by dopamine. ${ }^{\mathbf{1 4 6}}$ Han's research team fabricated tissue adhesive nanosheet using PDA. Catechol groups were oxidized and embedded within the sheets which contain free catechol functional groups, and acrylamide was allowed to interact with the sheets via free radical polymerization. They showed that the adhesives had good adhesive strengths of $120,80.8,80.7$, and $28.5 \mathrm{kPa}$ on glass, Ti, polyethylene ( $\mathrm{PE})$, and porcine skin, respectively. They demonstrated durable adhesiveness to both hydrophilic and hydrophobic substrates. They also provided repeatable adhesiveness onto skin over 20 cycles of peeling off. However the adhesive sheet did not adhere in a wet environment. ${ }^{\mathbf{1 4 7}}$

The major problem of applying adhesives in a form of film or sheet and patch to the wound site is mass production. The fabrication methods are complicated in some cases and need specific devices and techniques. However, this form of adhesives is able to be used in high tension sites of body because of high mechanical strength. ${ }^{128,135,148}$

\section{Evaluation of tissue adhesives}

\subsection{Macroscopic approaches}

The tissue adhesives were usually characterized in terms of their mechanical strength, biocompatibility and biodegradability.

Different methods have been used to evaluate the adhesive and cohesion strengths of the tissue adhesives using compression and tensile tests, lap shear and rheological analysis. Lap shear test can be conducted to evaluate the shear strength and modulus required to detach two materials of the same or different compositions adhered to each other by an adhesive. It can determine the adhesion strength at the interface in both wet and dry conditions. The type and thickness of the adhesives influence the lap shear strengths. In lap shear analyzes, the failure strain in tension for brittle adhesives ranged from $1.3 \%$ to $44 \%$ for the most ductile adhesives. ${ }^{100,149}$ Studies showed that the strength of lap-joint enhances when the bond line becomes thinner, thickness minimizes or in tough adhesives. The highest strength was obtained for bond lines in the range of 0.05 to $0.5 \mathrm{~mm}$. The adhesive strength may also be affected by other parameters, such as the type of loading including shear, peel, or cleavage, the adherent elastic or plastic behavior, and the adhesive ductility or brittleness. For instance, the failure load enhanced as the bond line became thicker in peel joints of ductile adhesives due to the adhesive load 
distribution over a larger area. ${ }^{\mathbf{1 4 9}}$ Dopamine incorporation in adhesive networks enhanced lap shear in wet condition. Stressstrain behavior in catechol-based tissue hydrogels showed up to 90\% strain without rupture. ${ }^{93}$ Real-time photo crosslinking rheology can be carried out with a UV light source or rheometer to show viscoelastic property. The samples were exposed to UV light for 2 minute after the first minute of data collection. $G^{\prime}$ and $G^{\prime \prime}$ demonstrate value of storage and loss modulus of adhesive, respectively. ${ }^{118}$ For all structures, when the storage modulus values were higher than the loss modulus values, it indicated that the adhesive was crosslinked covalently. ${ }^{100}$ The importance of sacrificial bond within the bioadhesive network was that it can dissipated energy of fracture under mechanical loads. ${ }^{\mathbf{1 1 5}}$ Universal Testing Machines (UTM) analysis has been used for measuring such mechanical properties as adhesion strength to the tissue, tensile and compressive strengths of adhesive materials. The primary and secondary geometry of adhesive species can be recorded before and after force loading, respectfully. ${ }^{91}$

To characterize biodegradability of tissue adhesives, in vitro and in vivo tests have been conducted. For degradation evaluation in vitro, the hydrogel is usually swollen in solution of PBS and sodium azide containing neutrophil elastase. After certain number of days, the degradation components in the solution can be evaluated. In vivo degradation characterization of samples can be conducted in mice or rats for a certain amount of time, then the samples be removed from the mice or rat for assessment. Depending on the type and application of the adhesives, the adhesive samples can be implanted into artery, skin or other tissues/organs.

\subsection{Microscopic approaches}

Microscopic assessments have been conducted to study chemical bonding, material surface morphology and roughness. Fourier transform infrared spectroscopy (FTIR) has been used to confirm the incorporation of amine/thiol groups into polymers. The DOPA dipping and dopamine crosslinking can be determined by specific peaks. FTIR peaks at the range of $1611 \mathrm{~cm}^{-1}$ suggest $\mathrm{N}-\mathrm{H}$ functional group in DOPA. ${ }^{94}$ In PEG and dopamine hydrogel, the peaks of $1000-1150 \mathrm{~cm}^{-1}, 2880 \mathrm{~cm}^{-1}$ and $1729 \mathrm{~cm}^{-1}$ assign to the ether, alkyl and carbonyl functional groups in PEG-dopamine chemical interaction, which is an indicating of crosslinking. ${ }^{95}{ }^{1} \mathrm{H}$ nuclear magnetic resonance spectroscopy ( ${ }^{1} \mathrm{H}$ NMR) and ultraviolet-visible (UV) spectrophotometer analysis have also been employed to characterize the structure. Interpolation of catechol to polymers is known by catechol concentration confirmed the degree of substitution of catechol. ${ }^{99}$ The chemical shift in ${ }^{1} \mathrm{H}$ NMR diagram provides information about the molecule structure. In a NMR spectrum, a peak at the 6.41-6.67 ranges shows the benzene ring protons. ${ }^{94}$ In addition, a study proved catechol chemical bonding through ${ }^{1} \mathrm{H}$ NMR characterization in which 6.6$6.8 \mathrm{ppm}$ signal demonstrated crosslinking between catechol and polylysine hydrogel. ${ }^{98}$ In this study, UV analysis represented catechol group moiety UV absorbance at $280 \mathrm{~nm} .{ }^{98} \mathrm{UV}$ is also used for the quantitative study of transition metal ions and highly conjugated organic compounds such as DOPA : $\mathrm{Fe}^{3+}$ and
DOPA : $\mathrm{IO}^{4-}$ in different $\mathrm{pH}$ conditions. ${ }^{\mathbf{8 8 , 1 1 5}}$ For an example, variation of $\mathrm{pH}$ changed the color of DOPA : $\mathrm{Fe}^{3+}$ to the green, purple and pink which demonstrated mono-, bis-, and triscomplexes, respectively. Covalent gelation in DOPA : $\mathrm{IO}^{4-}$ and noncovalent reaction in DOPA : $\mathrm{Fe}^{3+}$ have been investigated by color changes using UV. ${ }^{\mathbf{1 1 5}}$

Surface chemistry of tissue adhesives can be characterized using X-ray photoelectron spectroscopy (XPS). The surface of tissue adhesives before and after modification can be investigated specifically in the experiments that dopamine and its derivatives are applied in the form of coatings or surface modification. The most significant feature of polydopamine is its ability to be coated onto almost any substrate, effectively serving as an adlayer to change the surface property of the coated substrate and allowing further functionalization. XPS characterization of the substrate surfaces after coating revealed the complete disappearance of original substrate signals. ${ }^{52}$

Atomic force microscopy (AFM) has been used to characterize the surface morphology of the adhesives films, sheets and patches. ${ }^{148}$ Higher surface roughness suggests enhanced surface area, thus more surface absorption. ${ }^{139}$ In nanofibrous structures, surface roughness is related to the available surface coated by polydopamine. ${ }^{138}$ In addition, the adhesive property of tissue adhesives can be evaluates by AFM force analysis method. The adhesive forces can be measured at nanoscale by allowing the contact between the AFM cantilever tip and the adhesive surface, followed by a forced separation of the tip from the surface upon retraction. The force required for separation can be used as an indicator for adhesive strength at the surface/ interface. ${ }^{58,150}$

As to the characterization of biocompatibility, primary in vitro tests like the methlythiazoletetrazolium (MTT) assay, hemostasis analysis and histological analysis provide functionality and biocompatibility evaluations. The viability of cells exposed to the tissue adhesive samples determines cytotoxicity, with cell viability less than $70 \%$ being considered as nonbiocompatible adhesives. ${ }^{\mathbf{9 4 , 1 5 1}}$ In some studies, quantitative real-time polymerase chain reaction (qRT-PCR) analysis was done to exhibit gene expression that assist in understanding cytotoxicity and cell viability. ${ }^{91}$

Despite of the benefits and advantages of tissue adhesives discussed in this review, dopamine based tissue adhesives do show some limitations including low mechanical strength under loading in hard tissues repair, ${ }^{\mathbf{1 4 4}}$ poor adhesion in highly wet environment in which co-closure with suture has been required. ${ }^{\mathbf{1 4 7}}$ It is still a challenge to develop a perfect bioadhesive that can adhere to tissues and stop bleeding completely, be biocompatible, non-toxic and biodegradable, be cost effective and simple to use in order to attract surgeons for applications in complicated surgical situations. Furthermore, the synthetic techniques are complex for the majority of mussel inspired tissue adhesives. ${ }^{152}$ Several strategies have been done in recent years to face the current demands in tissue gluing; however, more characterizations and different side effects assessment in short or long terms use are needed. ${ }^{144}$ 


\section{Conclusion}

The use of traditional methods for wound closure like sutures, staples and flaps has certain disadvantages, particularly for delicate and vulnerable tissues. Tissue adhesives have been identified as a promising alternative for these purposes. They can also be used in combination with sutures in highly wet environment when the tissue glue by itself cannot hold tissues together. To tackle the problem of wet adhesion, dopaminebased adhesives in recently years have attracted extensive interests as a promising material to improve wet adhesive performance. Tissue adhesives also have applications in hard tissue gluing, drug or cell delivery systems and cell culture. Their potential in improvement of cell adhesion onto the surfaces is undeniable.

This review summarizes the recent advances in dopaminebased tissue adhesives that have been employed in biomedical applications. Different formats of dopamine-based adhesives, including glues, hydrogels and films used in the development of tissue adhesives with robust adhesiveness and excellent mechanical properties in biological and physiological environments, have been extensively explained. Tissue adhesives have potential to be grafted or added to different types of polymers, synthetic or natural, in order to provide various functionalities. In addition, tissue adhesives evaluation methods from both microscopic and macroscopic perspectives are discussed.

The biomimetic tissue adhesives are a rapidly expanding research field. Future work on the dopamine-based adhesives is expected to lead to the development of adhesive materials with excellent tissue compatibility, predictable biodegradability, appropriate mechanical properties and high wet adhesive capacities. Extra features such as self-healing, environmental responsive and loading of bioactive molecules, can be introduced to the adhesive materials to enhance their performance for different biomedical applications. The mechanisms of the outstanding adhesive capacity of dopamine or DOPA based materials have not been fully understand and therefore need further investigation. Other chemical, physical or surface characteristics of adhesive materials that may have an impact on their adhesive capacities are also worth extra research efforts.

\section{Conflicts of interest}

We confirm that none of the authors have any competing interests in the manuscript.

\section{List of abbreviations}

$\begin{array}{ll}\text { ECM } & \text { Extracellular matrix } \\ \text { AFM } & \text { Atomic force microscopy } \\ \text { ALP } & \text { Alkaline phosphatase } \\ \text { APE } & \text { Pentaerythritol triallyl ether } \\ \text { AspAm } & \text { Aspartamide } \\ \text { CA } & \text { Citric acid } \\ \text { cPEG } & \text { Catechol-terminated PEG } \\ \text { DAm } & \text { Dopamine acrylamide }\end{array}$

\begin{tabular}{|c|c|}
\hline DDS & Drug delivery system \\
\hline DMA & Dopamine methacrylate \\
\hline DOPA & 3,4-Dihydroxyphenylalanine \\
\hline ECM & Extra cellular matrix \\
\hline FDA & Food and drug administration \\
\hline FTIR & Fourier transform infrared spectroscopy \\
\hline GRF & Gelatin-resorcinol-formaldehyde \\
\hline GRFG & Gelatin-resorcinol-formaldehyde-glutaraldehyde \\
\hline HA & Hyaluronic acid \\
\hline HCA & Hydrocaffeic acid \\
\hline HEAA & 2-Hydroxyethyl acrylamide \\
\hline JKR & Johnson-Kendall-Roberts \\
\hline LbL & Layer by layer \\
\hline MEA & Methacryloyl chloride \\
\hline Mefp's & Mytilus edulis foot proteins \\
\hline MTT & Methlythiazoletetrazolium \\
\hline${ }^{1} \mathrm{H}$ NMR & ${ }^{1} \mathrm{H}$ nuclear magnetic resonance spectroscopy \\
\hline PAAm & Polyacrylamide \\
\hline PAE & $\operatorname{Poly}(\beta$-amino ester $)$ \\
\hline PCL & Polycaprolactone \\
\hline PDA & Polydopamine-co-acrylate \\
\hline PEG & Polyethylene glycol \\
\hline PEGDM & Dopamine-modified poly(ethylene glycol) \\
\hline PEI & Poly(ethylene imine) \\
\hline PEO & Poly(ethylene oxide) \\
\hline PEU & Poly(ester urea) \\
\hline PHS & Poly(parahydroxylstyrene) \\
\hline PNIPAM & Poly $(N$-isopropylacrylamide $)$ \\
\hline HD & Hexamethylenediamine \\
\hline PL & $\varepsilon$-Polylysine \\
\hline PLL & Poly(L-lysine) \\
\hline PMA & Poly(methacrylic acid) \\
\hline PPO & Poly(propylene oxide) \\
\hline PU & Polyurethanes \\
\hline HEPES & 4-(2-Hydroxyethyl)-1-piperazineethanesulfonic acid \\
\hline PHS & Poly(parahydroxylstyrene) \\
\hline PLCL & Poly(lactide-co-caprolactone) \\
\hline RGO & Reduced graphene oxide \\
\hline GO & Graphene oxide \\
\hline $\mathrm{PE}$ & Polyethylene \\
\hline QCM & Quartz crystal microbalance \\
\hline qRT-PCR & Quantitative real-time polymerase chain reaction \\
\hline RAFT & $\begin{array}{l}\text { Reversible addition fragmentation chain transfer } \\
\text { polymerization }\end{array}$ \\
\hline RGD & Arginine_glycine_aspartic acid \\
\hline PSI & Polysuccinimide \\
\hline SFA & Surface forces apparatus \\
\hline UTM & Universal testing machine \\
\hline UV & Ultraviolet-visible \\
\hline XPS & X-ray photoelectron spectroscopy \\
\hline
\end{tabular}

\section{Acknowledgements}

The authors want to acknowledge the support from NSERC Natural Sciences and Engineering Research Council of Canada (NSERC) Discovery grant. Authors would like to thank Mohammadreza Kazemian for his contribution in figures preparation. 


\section{References}

1 S. L. Basha, et al., Randomized controlled trial of wound complication rates of subcuticular suture vs staples for skin closure at cesarean delivery, Am. J. Obstet. Gynecol., 2010, 203(3), 285.

2 R. T. Forsch, Essentials of skin laceration repair, Am. Fam. Physician, 2008, 78(8), 945-951.

3 P. Heger, et al., Aktuelle Studienlage zum Bauchdeckenverschluss, Der Chirurg, 2016, 87(9), 737-743.

4 A. Islam and A. Ehsan, Comparison of suture material and technique of closure of subcutaneous fat and skin in caesarean section, N. Am. J. Med. Sci., 2011, 3(2), 85.

5 P. Sergeant, et al., Needle-to-suture ratio, as well as suture material, impacts needle-hole bleeding in vascular anastomoses, Interact. Cardiovasc. Thorac. Surg., 2016, 22(6), 813-816.

$6 \mathrm{~N} . \mathrm{Xu}$, et al., Light-activated sealing of skin wounds, Lasers Surg. Med., 2015, 47(1), 17-29.

7 J. Hochberg, K. M. Meyer and M. D. Marion, Suture choice and other methods of skin closure, Surg. Clin. North Am., 2009, 89(3), 627-641.

8 A. Duarte, et al., Surgical adhesives: systematic review of the main types and development forecast, Prog. Polym. Sci., 2012, 37(8), 1031-1050.

9 A. Lauto, D. Mawad and L. J. R. Foster, Adhesive biomaterials for tissue reconstruction, J. Chem. Technol. Biotechnol., 2008, 83(4), 464-472.

10 P. Coulthard, et al., Tissue adhesives for closure of surgical incisions, The Cochrane Library, 2010.

11 C. Yag-Howard, Sutures, needles, and tissue adhesives: a review for dermatologic surgery, Dermatol. Surg., 2014, 40, S3-S15.

12 A. L. Tajirian and D. J. Goldberg, A review of sutures and other skin closure materials, J. Cosmet. Laser Ther., 2010, 12(6), 296-302.

13 M. Rahimnejad, S. Derakhshanfar and W. Zhong, Biomaterials and tissue engineering for scar management in wound care, Burns \& Trauma, 2017, 5(1), 4.

$14 \mathrm{~S}$. Delibegović, et al., Tissue reaction to absorbable endoloop, nonabsorbable titanium staples, and polymer Hem-o-lok clip after laparoscopic appendectomy, J. Soc. Laparoendosc. Surg., 2011, 15(1), 70.

15 W. D. Spotnitz and S. G. Burks, Hemostats, sealants, and adhesives II: update as well as how and when to use the components of the surgical toolbox, Clin. Appl. Thromb./ Hemostasis, 2010, 497-514.

16 K. Yoshihara, et al., Nano-controlled molecular interaction at adhesive interfaces for hard tissue reconstruction, Acta Biomater., 2010, 6(9), 3573-3582.

17 P. Spencer, et al., Adhesive/dentin interface: the weak link in the composite restoration, Ann. Biomed. Eng., 2010, 38(6), 1989-2003.

18 M. Cardoso, et al., Current aspects on bonding effectiveness and stability in adhesive dentistry, Aust. Dent. J., 2011, 56(s1), 31-44.
19 A. Nordberg, et al., Highly adhesive phenolic compounds as interfacial primers for bone fracture fixations, ACS Appl. Mater. Interfaces, 2010, 2(3), 654-657.

20 H.-S. Jeong, et al., Use of fibrin glue for open comminuted nasal bone fractures, J. Craniofac. Surg., 2010, 21(1), 75-78.

$21 \mathrm{~J}$. Simson, et al., An orthopedic tissue adhesive for targeted delivery of intraoperative biologics, J. Orthop. Res., 2013, 31(3), 392-400.

22 J. H. Ryu, et al., Catechol-functionalized chitosan/pluronic hydrogels for tissue adhesives and hemostatic materials, Biomacromolecules, 2011, 12(7), 2653-2659.

23 N. Lang, et al., A blood-resistant surgical glue for minimally invasive repair of vessels and heart defects, Sci. Transl. Med., 2014, 6(218), 218 ra6.

24 H. C. Park, et al., Tissue adhesives in ocular surgery, Expert Rev. Ophthalmol., 2014, 631-655.

25 A. D. Mackeen, V. Berghella, and M. L. Larsen, Techniques and materials for skin closure in caesarean section, The Cochrane Library, 2012.

26 N. Annabi, et al., Surgical materials: current challenges and nano-enabled solutions, Nano Today, 2014, 9(5), 574-589.

27 B. Sharma, et al., Human cartilage repair with a photoreactive adhesive-hydrogel composite, Sci. Transl. Med., 2013, 5(167), 167ra6.

28 S. Vyas, S. Kamdar and P. Vyas, Tissue adhesives in ophthalmology, J. Clin. Ophthalmol. Res., 2013, 1(2), 107112.

$29 \mathrm{~W}$. Chen, et al., A mussel-inspired poly ( $\gamma$-glutamic acid) tissue adhesive with high wet strength for wound closure, J. Mater. Chem. B, 2017, 5(28), 5668-5678.

$30 \mathrm{~K}$. A. Vakalopoulos, et al., Tissue adhesives in gastrointestinal anastomosis: a systematic review, J. Surg. Res., 2013, 180(2), 290-300.

31 A. Sosnik, J. das Neves and B. Sarmento, Mucoadhesive polymers in the design of nano-drug delivery systems for administration by non-parenteral routes: a review, Prog. Polym. Sci., 2014, 39(12), 2030-2075.

32 P. P. Spicer and A. G. Mikos, Fibrin glue as a drug delivery system, J. Controlled Release, 2010, 148(1), 49-55.

33 S. H. Ku, J. S. Lee and C. B. Park, Spatial control of cell adhesion and patterning through mussel-inspired surface modification by polydopamine, Langmuir, 2010, 26(19), 15104-15108.

$34 \mathrm{~K}$. Sun, et al., Using self-polymerized dopamine to modify the antifouling property of oligo (ethylene glycol) selfassembled monolayers and its application in cell patterning, Langmuir, 2011, 27(10), 5709-5712.

$35 \mathrm{H}$. J. Kim, et al., Mussel adhesion-employed waterimmiscible fluid bioadhesive for urinary fistula sealing, Biomaterials, 2015, 72, 104-111.

36 Y. Ai, et al., Study on the synthesis and properties of mussel mimetic poly (ethylene glycol) bioadhesive, J. Photochem. Photobiol., B, 2013, 120, 183-190.

37 Y. Akdogan, et al., Intrinsic Surface-Drying Properties of Bioadhesive Proteins, Angew. Chem., 2014, 126(42), 1143511438. 
38 B.-H. Choi, et al., Highly purified mussel adhesive protein to secure biosafety for in vivo applications, Microb. Cell Fact., 2014, 13(1), 1.

39 N. Hiraishi, et al., Mussel mimetic, bioadhesive polymers from plant derived materials, J. Investig. Clin. Dent., 2015, 6(1), 59-62.

40 N. Bandara, H. Zeng and J. Wu, Marine mussel adhesion: biochemistry, mechanisms, and biomimetics, J. Adhes. Sci. Technol., 2013, 27(18-19), 2139-2162.

41 S. Ornes, Mussels' sticky feet lead to applications, Proc. Natl. Acad. Sci. U. S. A., 2013, 110(42), 16697-16699.

42 K. A. Bielefeld, S. Amini-Nik and B. A. Alman, Cutaneous wound healing: recruiting developmental pathways for regeneration, Cell. Mol. Life Sci., 2013, 70(12), 2059-2081.

43 M. C. Bloemen, et al., Prevention and curative management of hypertrophic scar formation, Burns, 2009, 35(4), 463-475.

$44 \mathrm{~J}$. Boateng and O. Catanzano, Advanced therapeutic dressings for effective wound healing-A Review, $J$. Pharm. Sci., 2015, 104(11), 3653-3680.

45 C. Profyris, C. Tziotzios and I. Do Vale, Cutaneous scarring: pathophysiology, molecular mechanisms, and scar reduction therapeutics: part I. The molecular basis of scar formation, J. Am. Acad. Dermatol., 2012, 66(1), 1-10.

46 C. Tziotzios, C. Profyris and J. Sterling, Cutaneous scarring: pathophysiology, molecular mechanisms, and scar reduction therapeutics: part II. Strategies to reduce scar formation after dermatologic procedures, J. Am. Acad. Dermatol., 2012, 66(1), 13-24.

47 M. Tschoi, E. A. Hoy and M. S. Granick, Skin flaps, Surg. Clin. North Am., 2009, 89(3), 643-658.

48 M. Matsuda, M. Inoue and T. Taguchi, Enhanced bonding strength of a novel tissue adhesive consisting of cholesteryl group-modified gelatin and disuccinimidyl tartarate, J. Bioact. Compat. Polym., 2012, 27(1), 31-44.

49 E. Y. Jeon, et al., Rapidly light-activated surgical protein glue inspired by mussel adhesion and insect structural crosslinking, Biomaterials, 2015, 67, 11-19.

$50 \mathrm{~A}$. Chambers and M. Scarci, Is skin closure with cyanoacrylate glue effective for the prevention of sternal wound infections?, Interact. Cardiovasc. Thorac. Surg., 2010, 10(5), 793-796.

$51 \mathrm{~J}$. W. Gooch, Biocompatible polymeric materials and tourniquets for wounds, Springer Science \& Business Media, 2010.

$52 \mathrm{H}$. Lee, et al., Mussel-inspired surface chemistry for multifunctional coatings, Science, 2007, 318(5849), 426-430.

53 B. P. Lee, et al., Mussel-inspired adhesives and coatings, Annu. Rev. Mater. Res., 2011, 41, 99.

54 S. C. Nicklisch and J. H. Waite, Mini-review: The role of redox in Dopa-mediated marine adhesion, Biofouling, 2012, 28(8), 865-877.

$55 \mathrm{~J}$. Yu, et al., Mussel protein adhesion depends on interprotein thiol-mediated redox modulation, Nat. Chem. Biol., 2011, 7(9), 588-590.

56 K. M. Gray, et al., Biomimetic fabrication of informationrich phenolic-chitosan films, Soft Matter, 2011, 7(20), 9601-9615.
57 C. Ghobril and M. Grinstaff, The chemistry and engineering of polymeric hydrogel adhesives for wound closure: a tutorial, Chem. Soc. Rev., 2015, 44(7), 1820-1835.

$58 \mathrm{H}$. Lee, B. P. Lee and P. B. Messersmith, A reversible wet/dry adhesive inspired by mussels and geckos, Nature, 2007, 448(7151), 338-341.

59 C. R. Matos-Pérez, J. D. White and J. J. Wilker, Polymer composition and substrate influences on the adhesive bonding of a biomimetic, cross-linking polymer, J. Am. Chem. Soc., 2012, 134(22), 9498-9505.

60 S. M. Kang, et al., One-step modification of superhydrophobic surfaces by a mussel-inspired polymer coating, Angew. Chem., Int. Ed., 2010, 49(49), 9401-9404.

61 S. Moulay, Dopa/catechol-tethered polymers: Bioadhesives and biomimetic adhesive materials, Polym. Rev., 2014, 54(3), 436-513.

62 M. Mehdizadeh and J. Yang, Design strategies and applications of tissue bioadhesives, Macromol. Biosci., 2013, 13(3), 271-288.

$63 \mathrm{~J}$. H. Waite, Mussel adhesion - essential footwork, J. Exp. Biol., 2017, 220(4), 517-530.

64 B. Krishnamoorthy, et al., Randomized prospective study comparing conventional subcuticular skin closure with Dermabond skin glue after saphenous vein harvesting, Ann. Thorac. Surg., 2009, 88(5), 1445-1449.

65 A. Dragu, et al., Foreign body reaction after usage of tissue adhesives for skin closure: a case report and review of the literature, Arch. Orthop. Trauma Surg., 2009, 129(2), 167169.

66 Y. S. Choi, et al., Recombinant mussel adhesive protein fp-5 (MAP fp-5) as a bulk bioadhesive and surface coating material, Biofouling, 2011, 27(7), 729-737.

67 T. Wang, J. Nie and D. Yang, Dextran and gelatin based photocrosslinkable tissue adhesive, Carbohydr. Polym., 2012, 90(4), 1428-1436.

68 L. Vollenweider, et al., Biomimetic adhesive coatings for soft tissue repair, 15th Int Coat Sci Technol Sym, 2010.

$69 \mathrm{H}$. Shao and R. J. Stewart, Biomimetic underwater adhesives with environmentally triggered setting mechanisms, Adv. Mater., 2010, 22(6), 729-733.

$70 \mathrm{H}$. Zhang, et al., A biomimetic hyperbranched poly (amino ester)-based nanocomposite as a tunable bone adhesive for sternal closure, J. Mater. Chem. B, 2014, 2(26), 4067-4071.

$71 \mathrm{H}$. Zhang, et al., Mussel-inspired hyperbranched poly (amino ester) polymer as strong wet tissue adhesive, Biomaterials, 2014, 35(2), 711-719.

$72 \mathrm{~J}$. Zhou, et al., Adhesion properties of catechol-based biodegradable amino acid-based poly (ester urea) copolymers inspired from mussel proteins, Biomacromolecules, 2014, 16(1), 266-274.

73 B. K. Ahn, et al., High-performance mussel-inspired adhesives of reduced complexity, Nat. Commun., 2015, 6, 8663.

74 S.-B. Lee, et al., Catechol-Functionalized Synthetic Polymer as a Dental Adhesive to Contaminated Dentin Surface for a Composite Restoration, Biomacromolecules, 2015, 16(8), 2265-2275. 
75 Y. Ji, et al., Mussel-inspired soft-tissue adhesive based on poly (diol citrate) with catechol functionality, J. Mater. Sci.: Mater. Med., 2016, 27(2), 1-9.

76 S. Hong, et al., Supramolecular Metallo-Bioadhesive for Minimally Invasive Use, Adv. Mater., 2016, 28(39), 86758680.

77 A. Kawabata, et al., A foreign body granuloma after the usage of polyglycolic acid mesh and fibrin glue for dural repair. A case report, J. Orthop. Sci., 2015, 22(2), 371-374.

$78 \mathrm{~L}$. Yu and J. Ding, Injectable hydrogels as unique biomedical materials, Chem. Soc. Rev., 2008, 37(8), 14731481.

79 C. T. Huynh, M. K. Nguyen and D. S. Lee, Injectable block copolymer hydrogels: achievements and future challenges for biomedical applications, Macromolecules, 2011, 44(17), 6629-6636.

80 M. D. Hager, et al., Self-Healing Materials, Adv. Mater., 2010, 22(47), 5424-5430.

81 L. Moroni and J. H. Elisseeff, Biomaterials engineered for integration, Mater. Today, 2008, 11(5), 44-51.

82 L. P. Bré, et al., Taking tissue adhesives to the future: from traditional synthetic to new biomimetic approaches, Biomater. Sci., 2013, 1(3), 239-253.

83 J. I. Paez, et al., Gauging and tuning cross-linking kinetics of catechol-PEG adhesives via catecholamine functionalization, Biomacromolecules, 2015, 16(12), 38113818.

84 J. Sedó, et al., Catechol-Based Biomimetic Functional Materials, Adv. Mater., 2013, 25(5), 653-701.

85 Y. Yang, et al., A biocompatible and functional adhesive amine-rich coating based on dopamine polymerization, $J$. Mater. Chem. B, 2015, 3(1), 72-81.

$86 \mathrm{~J}$. H. An, et al., Surface modification using bio-inspired adhesive polymers based on polyaspartamide derivatives, Polym. Int., 2011, 60(11), 1581-1586.

$87 \mathrm{~J} . \mathrm{Xu}$, et al., Mollusk glue inspired mucoadhesives for biomedical applications, Langmuir, 2012, 28(39), 1401014017.

88 Y. C. Choi, et al., Human gelatin tissue-adhesive hydrogels prepared by enzyme-mediated biosynthesis of DOPA and Fe 3+ ion crosslinking, J. Mater. Chem. B, 2014, 2(2), 201-209.

$89 \mathrm{~W}$. Zhu, et al., A mussel-inspired double-crosslink tissue adhesive on rat mastectomy model: seroma prevention and in vivo biocompatibility, J. Surg. Res., 2017, 215, 173182.

90 C. Fan, et al., A mussel-inspired double-crosslinked tissue adhesive intended for internal medical use, Acta Biomater., 2016, 33, 51-63.

91 J. Shin, et al., Tissue Adhesive Catechol-Modified Hyaluronic Acid Hydrogel for Effective, Minimally Invasive Cell Therapy, Adv. Funct. Mater., 2015, 25(25), 3814-3824.

92 C. E. Brubaker, et al., Biological performance of musselinspired adhesive in extrahepatic islet transplantation, Biomaterials, 2010, 31(3), 420-427.

93 D. G. Barrett, G. G. Bushnell and P. B. Messersmith, Mechanically robust, negative-swelling, mussel-inspired tissue adhesives, Adv. Healthcare Mater., 2013, 2(5), 745755.

$94 \mathrm{C}$. Li, et al., Photocrosslinkable bioadhesive based on dextran and PEG derivatives, Mater. Sci. Eng. C, 2014, 35, 300-306.

95 Y. Liu, et al., Injectable dopamine-modified poly (ethylene glycol) nanocomposite hydrogel with enhanced adhesive property and bioactivity, ACS Appl. Mater. Interfaces, 2014, 6(19), 16982-16992.

96 S. Skelton, et al., Biomimetic adhesive containing nanocomposite hydrogel with enhanced materials properties, Soft Matter, 2013, 9(14), 3825-3833.

97 C. L. Jenkins, H. J. Meredith and J. J. Wilker, Molecular weight effects upon the adhesive bonding of a mussel mimetic polymer, ACS Appl. Mater. Interfaces, 2013, 5(11), 5091-5096.

98 Y. Zhou, et al., Rapid gelling chitosan/polylysine hydrogel with enhanced bulk cohesive and interfacial adhesive force: mimicking features of epineurial matrix for peripheral nerve anastomosis, Biomacromolecules, 2016, 17(2), 622-630.

99 K. A. Burke, D. C. Roberts and D. L. Kaplan, Silk Fibroin Aqueous-Based Adhesives Inspired by Mussel Adhesive Proteins, Biomacromolecules, 2015, 17(1), 237-245.

100 Y. Li, et al., Gelatin Microgel Incorporated Poly (Ethylene Glycol)-based Bioadhesive with Enhanced Adhesive Property and Bioactivity, ACS Appl. Mater. Interfaces, 2016, 8(19), 11980-11989.

$101 \mathrm{~J}$. Guo, et al., Synthesis and characterization of antibacterial and anti-fungal citrate-based mussel-inspired bioadhesives, Biomaterials, 2016, 85, 204-217.

$102 \mathrm{~J}$. Guo, et al., Click chemistry improved wet adhesion strength of mussel-inspired citrate-based antimicrobial bioadhesives, Biomaterials, 2017, 112, 275-286.

103 Y. Ji, et al., Mussel-inspired soft-tissue adhesive based on poly (diol citrate) with catechol functionality, J. Mater. Sci.: Mater. Med., 2016, 27(2), 30.

104 B. Yang, et al., Facilely prepared inexpensive and biocompatible self-healing hydrogel: a new injectable cell therapy carrier, Polym. Chem., 2012, 3(12), 3235-3238.

105 B. K. Ahn, et al., Surface-initiated self-healing of polymers in aqueous media, Nat. Mater., 2014, 13(9), 867-872.

106 N. Holten-Andersen, et al., pH-induced metal-ligand crosslinks inspired by mussel yield self-healing polymer networks with near-covalent elastic moduli, Proc. Natl. Acad. Sci. U. S. A., 2011, 108(7), 2651-2655.

107 M. Krogsgaard, et al., Self-healing mussel-inspired multipH-responsive hydrogels, Biomacromolecules, 2013, 14(2), 297-301.

$108 \mathrm{~L}$. He, et al., pH responsive self-healing hydrogels formed by boronate-catechol complexation, Chem. Commun., 2011, 47(26), 7497-7499.

109 M. Vatankhah-Varnoosfaderani, et al., Rapid self-healing and triple stimuli responsiveness of a supramolecular polymer gel based on boron-catechol interactions in a novel water-soluble mussel-inspired copolymer, Polym. Chem., 2014, 5(2), 512-523. 
110 M. Shan, et al., A pH, glucose, and dopamine tripleresponsive, self-healable adhesive hydrogel formed by phenylborate-catechol complexation, Polym. Chem., 2017, 8(19), 2997-3005.

111 S. Hong, et al., Hyaluronic Acid Catechol: A Biopolymer Exhibiting a pH-Dependent Adhesive or Cohesive Property for Human Neural Stem Cell Engineering, Adv. Funct. Mater., 2013, 23(14), 1774-1780.

112 P. Sun, et al., Facile Preparation of Mussel-Inspired Polyurethane Hydrogel and Its Rapid Curing Behavior, ACS Appl. Mater. Interfaces, 2014, 6(15), 12495-12504.

$113 \mathrm{M}$. Cencer, et al., Effect of $\mathrm{pH}$ on the rate of curing and bioadhesive properties of dopamine functionalized poly (ethylene glycol) hydrogels, Biomacromolecules, 2014, 15(8), 2861-2869.

114 M. Cencer, et al., Effect of nitro-functionalization on the cross-linking and bioadhesion of biomimetic adhesive moiety, Biomacromolecules, 2014, 16(1), 404-410.

115 B. J. Kim, et al., Mussel-mimetic protein-based adhesive hydrogel, Biomacromolecules, 2014, 15(5), 1579-1585.

116 B. Yang, et al., In Vivo Residue-Specific Dopa-Incorporated Engineered Mussel Bioglue with Enhanced Adhesion and Water Resistance, Angew. Chem., Int. Ed., 2014, 53(49), 13360-13364.

117 Y. Lee, et al., Thermo-sensitive, injectable, and tissue adhesive sol-gel transition hyaluronic acid/pluronic composite hydrogels prepared from bio-inspired catecholthiol reaction, Soft Matter, 2010, 6(5), 977-983.

$118 \mathrm{H}$. Zhang, et al., On-demand and negative-thermo-swelling tissue adhesive based on highly branched ambivalent PEGcatechol copolymers, J. Mater. Chem. B, 2015, 3(31), 64206428.

119 B. Laulicht, et al., Bioinspired Bioadhesive Polymers: DopaModified Poly (acrylic acid) Derivatives, Macromol. Biosci., 2012, 12(11), 1555-1565.

120 C. J. Kastrup, et al., Painting blood vessels and atherosclerotic plaques with an adhesive drug depot, Proc. Natl. Acad. Sci. U. S. A., 2012, 109(52), 21444-21449.

121 D. E. Fullenkamp, et al., Mussel-inspired silver-releasing antibacterial hydrogels, Biomaterials, 2012, 33(15), 37833791.

122 S. Lim, et al., Bioadhesive Nanoaggregates Based on Polyaspartamide-g-C18/DOPA for Wound Healing, Biomacromolecules, 2017, 18(8), 2402-2409.

123 C. Gong, et al., Injectable dopamine-modified poly ( $\alpha, \beta$ aspartic acid) nanocomposite hydrogel as bioadhesive drug delivery system, J. Biomed. Mater. Res., Part A, 2017, 105(4), 1000-1008.

124 L. S. M. Teixeira, et al., Enzyme-catalyzed crosslinkable hydrogels: emerging strategies for tissue engineering, Biomaterials, 2012, 33(5), 1281-1290.

125 C. E. Brubaker and P. B. Messersmith, Enzymatically degradable mussel-inspired adhesive hydrogel, Biomacromolecules, 2011, 12(12), 4326-4334.

126 B. P. Lee and S. Konst, Novel hydrogel actuator inspired by reversible mussel adhesive protein chemistry, Adv. Mater., 2014, 26(21), 3415-3419.
127 A. B. South and L. A. Lyon, Autonomic Self-Healing of Hydrogel Thin Films, Angew. Chem., 2010, 122(4), 779-783.

128 W. Liu, S. Thomopoulos and Y. Xia, Electrospun nanofibers for regenerative medicine, Adv. Healthcare Mater., 2012, 1(1), 10-25.

$129 \mathrm{H}$. Zeng, et al., Strong reversible Fe3+-mediated bridging between dopa-containing protein films in water, Proc. Natl. Acad. Sci. U. S. A., 2010, 107(29), 12850-12853.

130 J. L. Murphy, et al., Adhesive performance of biomimetic adhesive-coated biologic scaffolds, Biomacromolecules, 2010, 11(11), 2976-2984.

131 B. J. Sparks, et al., Mussel-inspired thiol-ene polymer networks: Influencing network properties and adhesion with catechol functionality, Chem. Mater., 2012, 24(18), 3633-3642.

132 K. Kim, et al., Bio-inspired catechol conjugation converts water-insoluble chitosan into a highly water-soluble, adhesive chitosan derivative for hydrogels and $\mathrm{LbL}$ assembly, Biomater. Sci., 2013, 1(7), 783-790.

133 D. X. Oh and D. S. Hwang, A biomimetic chitosan composite with improved mechanical properties in wet conditions, Biotechnol. Prog., 2013, 29(2), 505-512.

134 Y. M. Shin, et al., Bio-inspired Immobilization of CellAdhesive Ligands on Electrospun Nanofibrous Patches for Cell Delivery, Macromol. Mater. Eng., 2013, 298(5), 555-564.

135 Y. Zhang, et al., Highly-branched poly (Nisopropylacrylamide) as a component in poly (dopamine) films, J. Phys. Chem. B, 2013, 117(36), 10504-10512.

136 L. Guo, et al., A mussel-inspired polydopamine coating as a versatile platform for the in situ synthesis of graphenebased nanocomposites, Nanoscale, 2012, 4(19), 5864-5867.

137 C. Cheng, et al., General and biomimetic approach to biopolymer-functionalized graphene oxide nanosheet through adhesive dopamine, Biomacromolecules, 2012, 13(12), 4236-4246.

138 L. Ge, et al., Polydopamine-coated paper-stack nanofibrous membranes enhancing adipose stem cells' adhesion and osteogenic differentiation, J. Mater. Chem. B, 2014, 2(40), 6917-6923.

139 A. I. Neto, et al., Nanostructured Polymeric Coatings Based on Chitosan and Dopamine-Modified Hyaluronic Acid for Biomedical Applications, Small, 2014, 10(12), 2459-2469.

$140 \mathrm{~J} . \mathrm{H}$. Ryu, et al., Bio-Inspired, Water-Soluble to Insoluble Self-Conversion for Flexible, Biocompatible, Transparent, Catecholamine Polysaccharide Thin Films, Adv. Funct. Mater., 2014, 24(48), 7709-7716.

$141 \mathrm{~J}$. Jiang, et al., Mussel-inspired nanofibrous sheet for suture-less stomach incision surgery, Chem. Commun., 2015, 51(41), 8695-8698.

142 Y. Zhang, et al., Mixed poly (dopamine)/poly (1lysine)(composite) coatings: from assembly to interaction with endothelial cells, Biomater. Sci., 2015, 3(8), 1188-1196.

143 M. M. Zieger, et al., Ultrathin Monomolecular Films and Robust Assemblies Based on Cyclic Catechols, Langmuir, 2017, 33(3), 670-679.

$144 \mathrm{~K}$. Olofsson, et al., Activated dopamine derivatives as primers for adhesive-patch fixation of bone fractures, $R S C$ Adv., 2016, 6(31), 26398-26405. 
145 F. Scognamiglio, et al., Enhanced bioadhesivity of dopaminefunctionalized polysaccharidic membranes for general surgery applications, Acta Biomater., 2016, 44, 232-242.

146 F. Scognamiglio, et al., Adhesive coatings based on melanin-like nanoparticles for surgical membranes, Colloids Surf., B, 2017, 155, 553-559.

147 L. Han, et al., Mussel-Inspired Adhesive and Tough Hydrogel Based on Nanoclay Confined Dopamine Polymerization, ACS Nano, 2017, 11(3), 2561-2574.

$148 \mathrm{~J}$. Longo, et al., Stable Bioactive Enzyme-Containing Multilayer Films Based on Covalent Cross-Linking from Mussel-Inspired Adhesives, Langmuir, 2015, 31(45), 12447-12454.
149 H. Yuk, et al., Tough bonding of hydrogels to diverse nonporous surfaces, Nat. Mater., 2016, 15(2), 190-196.

150 M. Xing, et al., Adhesion force studies of nanofibers and nanoparticles, Langmuir, 2010, 26(14), 11809-11814.

151 M. Mehdizadeh, et al., Injectable citrate-based musselinspired tissue bioadhesives with high wet strength for sutureless wound closure, Biomaterials, 2012, 33(32), 7972-7983.

152 P. Kord Forooshani and B. P. Lee, Recent approaches in designing bioadhesive materials inspired by mussel adhesive protein, J. Polym. Sci., Part A: Polym. Chem., 2016, 55(1), 9-33. 